\title{
Temperature Control of PEMFC Stack Based on BP Neural Network Guo $\mathrm{Li}^{1,}$, , Yang $\mathrm{Li}^{2, \mathrm{~b}}$ \\ ${ }^{1}$ Automation School, University of Science and Technology in Beijing, Beijing, 100083, China \\ 2 Automation School, University of Science and Technology in Beijing, Beijing,100083, China aguo6396@sina.com, ${ }^{\mathrm{b}}$ liyang_chn@163.com
}

Keywords: PEM fuel cell; temperature control; BP neural network

\begin{abstract}
PEMFC (proton exchange membrane fuel cell) Stack temperature control system is a strong-coupling system with characteristics of time-change, long-hysteresis, uncertainty and nonlinear. The dissertation studied PEMFC stack temperature control used by BP neural network controller which has a good control quality and a low degree of demand for model. In the Matlab platform, the model of the electric reactor temperature control system is established and compared with the traditional PID. It has been proved that BP neural network control system has very good robustness and control quality and it meet the demand of PEMFC stack temperature control system.
\end{abstract}

\section{Introduction}

In various types of fuel cells, PEMFC has many unique advantages, and has become the most rapidly developing fuel cell in recent years. In the past, the research on PEMFC has focused on the improvement of the chemical properties of the electrolyte membrane, the decrease of the platinum catalyst, the change of the structure of the membrane electrode, the enhancement of anti $\mathrm{CO}$, the ability and the preparation technology, with the advent of commercialization, the control technology of PEMFC becomes an important issue.

\section{PEMFC Stack Temperature Control Model}

The overall temperature and temperature distribution in the PEMFC Stack is an important control object of the fuel cell test system, and also the most important research object in the PEMFC Stack.

The heat generated by the electrochemical reaction can be approximated as:

$$
\Delta Q=I_{\text {out }}\left(V_{\text {equ }}-V_{\text {out }}\right)
$$

$\mathrm{V}_{\text {equ }}$ is the equivalent single battery output voltage when the chemical energy is converted to heat energy. The heat taken away by the Stack cooling is:

$$
\Delta Q_{\text {con }}=c \rho V_{\text {cool }} \cdot \Delta T_{\text {cool }}
$$

$V_{\text {cool }}$ is the flow of cooling fluid in the unit time, $\Delta T_{\text {cool }}$ is the temperature difference in the cycle. The temperature model of the PEMFC Stack is:

$$
T(k+1)=\phi\left[V_{H}(k), V_{O}(k), V_{W}(k), T(k)\right]
$$

$V_{H}(k), V_{O}(k), V_{W}(k)$ are hydrogen, oxygen and coolant flow; $T(k)$ is the working temperature of the stack. After identification and simplification, the n-order plus pure delay model are obtained:

$$
G(z)=\frac{Y(s)}{U(s)}=\frac{\alpha e^{-\tau s}}{\left(b_{1} s+1\right)\left(b_{2} s+1\right) \cdots\left(b_{n} s+1\right)}
$$


$Y(s)$ is a power reactor temperature, $U(s)$ is a multi-input parameter, Including hydrogen and oxygen flow and coolant flow.

\section{Structure Design of Control System}

Analysis of the mathematical model of the temperature control system, BP neural network is used to construct the PID controller, as shown in Fig.1.

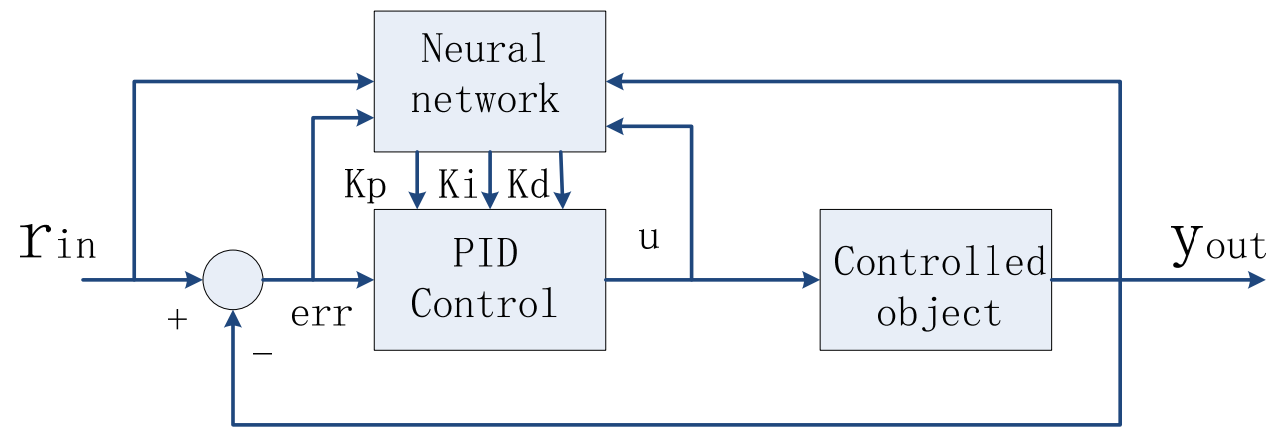

Fig.1 PEM neural network stack temperature control system

PID Control Algorithm for Integral Separation. Fuel cell temperature control is a slow process, which has long-hysteresis. In order to prevent the integral term in the controller, the PID control algorithm with integral separation is adopted, which can be expressed as:

$$
u(k)=k_{p}(\operatorname{err}(k)-\operatorname{err}(k-1))+\beta k_{i} \operatorname{err}(k) T_{s}+\frac{k_{d}}{T_{s}}(\operatorname{err}(k)-2 \operatorname{err}(k-1)+\operatorname{err}(k-2))
$$

$T_{s}$ is the actual sampling rate, $\beta$ is the integral term of the switching coefficient. $\beta$ values are as follows:

$$
\beta= \begin{cases}1 & \text { when }|\operatorname{err}(\mathrm{k})| \leq \varepsilon \\ 2 & \text { when }|\operatorname{err}(\mathrm{k})|>\varepsilon\end{cases}
$$

PID Control Algorithm for BP Neural Network. $\boldsymbol{k}_{\boldsymbol{p}}, \boldsymbol{k}_{\boldsymbol{i}}$ and $\boldsymbol{k}_{\boldsymbol{d}}$ are considered to be dependent on the adjustable coefficient of the system running state. The Eq.5 is described as:

$$
u(k)=f\left\{\operatorname{err}(k), \operatorname{err}(k-1), \operatorname{err}(k-2), k_{p}, k_{i}, k_{d}\right\}
$$

So the operating parameters and the state of the whole control system can be adjusted by BP neural network to find an optimal control law.

\section{Simulation of Control System}

In this paper, the controlled object is the n-order plus pure delay model. We select the parameters of the transfer function of the class 1kw PEMFC Stack is: $a=0.7, \tau=5, b_{1}=5.5, b_{2}=4.5, b_{3}=3.5$ $, b_{4}=2.5, b_{5}=1.5$.

Simulation Results of BP Neural Network PID Control. BP neural network control is performed under the same experimental conditions. The three layer BP neural network is used for 4-5-3, the weighted coefficient matrix of the hidden layer to the output layer is $\boldsymbol{w}_{\boldsymbol{i}}(\mathbf{5} \times \mathbf{4})$. The connection weight matrix of the input layer to the hidden layer is $\boldsymbol{w}_{\boldsymbol{o}}(\mathbf{3} \times \mathbf{5})$. The initial value is:

$$
w_{o}=\left[\begin{array}{ccccc}
0.7576 & 0.2616 & 0.5820 & -0.1416 & -0.1325 \\
-0.1146 & 0.2949 & 0.8352 & 0.2205 & 0.4508 \\
0.7201 & 0.4566 & 0.7672 & 0.4962 & 0.3632
\end{array}\right]
$$




$$
w_{i}=\left[\begin{array}{cccc}
-0.6394 & -0.2696 & -0.3756 & -0.7023 \\
-0.8603 & -0.2013 & -0.5024 & -0.2596 \\
-1.0749 & 0.5543 & -1.6820 & -0.2596 \\
-0.3625 & -0.0724 & -0.6463 & -0.2859 \\
0.1425 & 0.0279 & -0.5406 & -0.7660
\end{array}\right]
$$

The convergence of the algorithm is very important and cannot be modified. They are the corresponding values after the initial PID parameters of the neural network. On this basis, when the running state of small amplitude change of the system, ensure the stability and convergence of the algorithm.

According to the results of many experiments show that, when the learning step $\eta$ between 0.005 0.03 values, momentum factor $\alpha$ between 0.6 0.9 values, is the most appropriate for the BP algorithm, so the values were 0.01 and 0.8 respectively. Simulation results are shown in Fig.2.

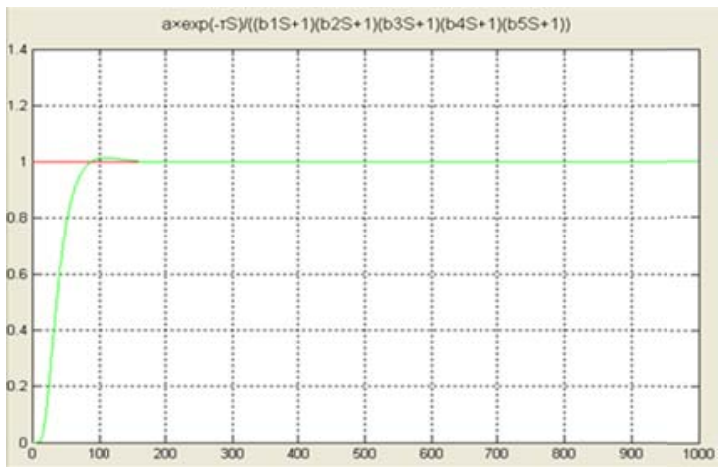

Fig.2 Neural network PID control simulation

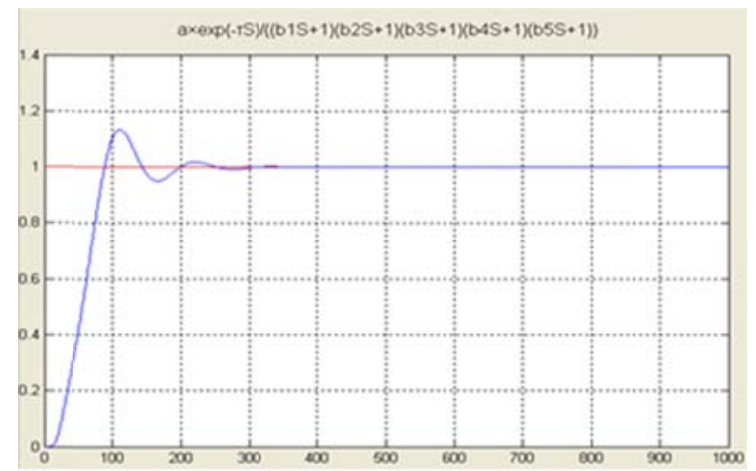

Fig.3 Integral separation PID control simulation

Simulation Results of Integral Separation PID Control. In this paper, a segmentation method is used to compare the traditional PID and neural network PID. Let $\boldsymbol{K}_{\boldsymbol{p}}=\mathbf{0 . 0 8}, \boldsymbol{K}_{\boldsymbol{i}}=\mathbf{0 . 0 6 5}, \boldsymbol{K}_{\boldsymbol{d}}=$ 0. 01. The simulation results are shown in Fig.3.

From Fig.2 and Fig.3 we can see, BP neural network PID rise time $t_{r} \approx 70 s$.Regulation time $t_{s} \approx 150 \mathrm{~s}$, overshoot $\sigma \% \approx 2 \%$. Integral separation PID rise time $t_{r} \approx 80 \mathrm{~s}$. Regulation time $t_{s} \approx 240 \mathrm{~s}$, overshoot $\sigma \% \approx 10 \%$. Obviously the superiority of PID algorithm in neural network is obvious.

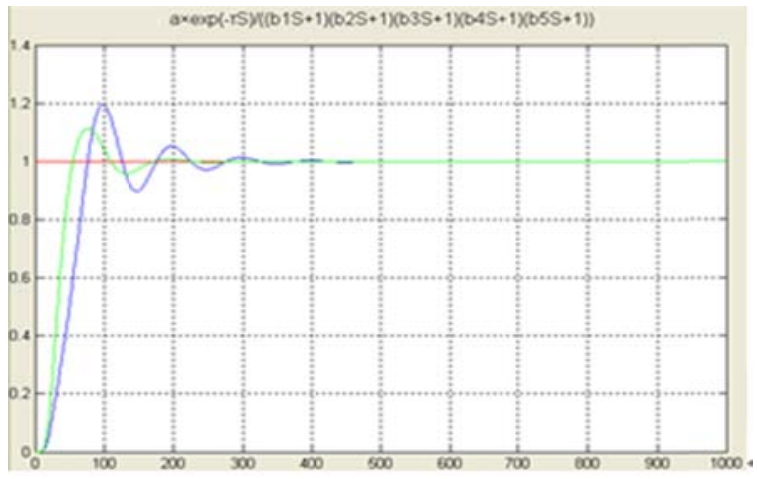

Fig.4 When the results of the increase

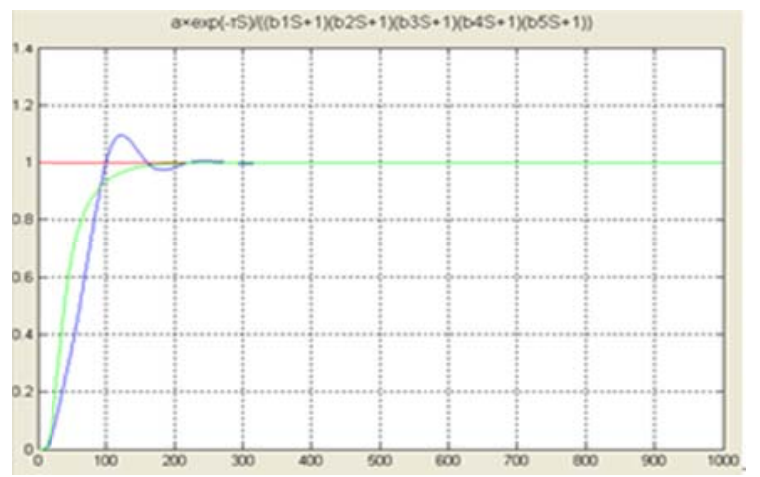

Fig. 5 When the resultsi of the reduction

Comparison of two Control Modes for Parameter Variation. When parameter a Changes, a: $\mathbf{0 . 7} \rightarrow \mathbf{0 . 8 6}$ is shown in Fig.4; a: $\mathbf{0 . 7} \rightarrow \mathbf{0 . 6}$ is shown in Fig.5. When the results of a increase, PID control rise timet $t_{\mathbf{r}} \approx \mathbf{7 0 s}$, regulation timet $\mathbf{s}_{\mathbf{s}} \approx \mathbf{3 0 0 s}$, overshoot $\boldsymbol{\sigma} \% \approx \mathbf{2 0} \%$;NNPID control rise timet $t_{\mathrm{r}} \approx \mathbf{5 0 s}$, regulation timet $\mathrm{t}_{\mathrm{s}} \approx \mathbf{1 8 0 \mathrm { s }}$ overshoot $\% \approx \mathbf{1 0} \%$. When the results of a reduction, PID control rise timet $t_{\mathbf{r}} \approx \mathbf{1 0 0 s}$, regulation timet $\mathbf{t}_{\mathbf{s}} \approx \mathbf{2 1 0 s}$, overshoot $\sigma \% \approx \mathbf{1 0} \%$; NNPID control rise timet $t_{\mathbf{r}} \approx \mathbf{2 0 0 s}$, regulation timet $\mathbf{s}_{\mathrm{s}} \approx \mathbf{1 8 0}$ overshoot $\boldsymbol{\sigma} \% \approx \mathbf{0} \%$.

Because of $b_{1}, b_{2}, b_{3}, b_{4}, b_{5}$ effect are the same, only choose $b_{1}$ parameter variation analysis. When parameter $b_{1}$ Changes, $b_{1}: 5.5 \rightarrow 9.5$ is shown in figure $6 ; b_{1}: 5.5 \rightarrow 2.3$ is shown in 
Fig.7. When the results of $b_{1}$ increase, PID control rise timet $\mathrm{r}_{\mathrm{r}} \approx 90 \mathrm{~s}$, regulation timet $\mathrm{s}_{\mathrm{s}} \approx 310 \mathrm{~s}$,

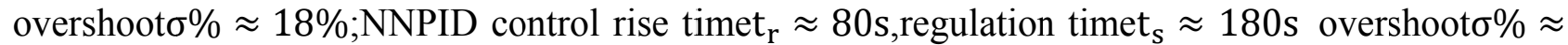
$6 \%$. When the results of $b_{1}$ reduction, PID control rise timet $_{\mathrm{r}} \approx 80 \mathrm{~s}$, regulation timet $\mathrm{s} \approx 180 \mathrm{~s}$, overshoot $\sigma \% \approx 10 \%$; NNPID control rise timet $\mathrm{r} \approx 100 \mathrm{~s}$, regulation timet ${ }_{\mathrm{s}} \approx 120 \mathrm{~s}$, overshoot $\sigma \% \approx 1 \%$.

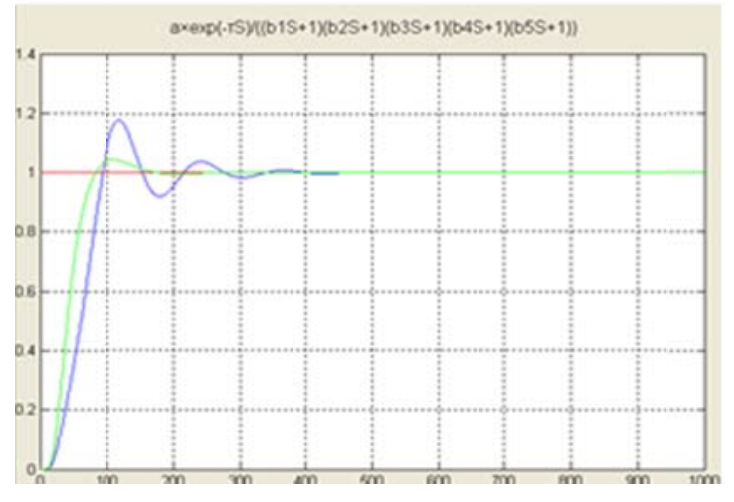

Fig.6 When the results of the $b_{1}$ increase

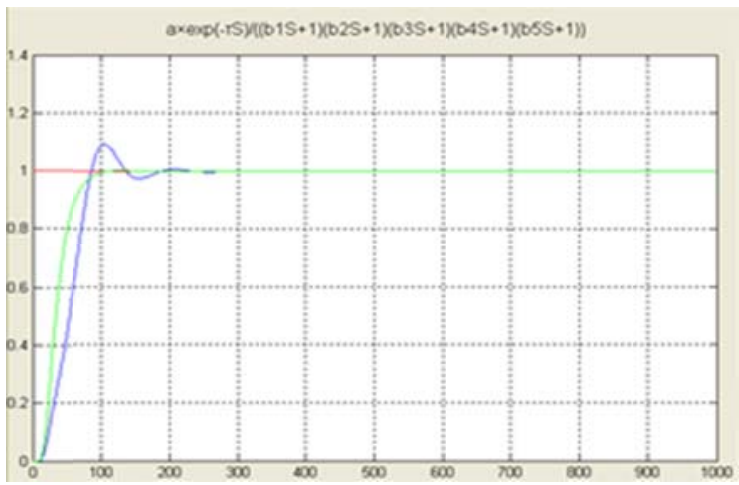

Fig. 7 When the results of the $b_{1}$ reduction

When parameter $\tau$ Changes, $\tau: 5 \rightarrow 7$ is shown in Fig.8; $\tau: 5 \rightarrow 2$ is shown in Fig.9. When the results of $\tau$ increase, PID control rise time $t_{r} \approx 90 s$, regulation time $\mathrm{t}_{\mathrm{s}} \approx 300 \mathrm{~s}$, overshoot $\sigma \% \approx 18 \%$; NNPID control rise time $t_{r} \approx 70 s$, regulation time $t_{s} \approx 180 \mathrm{~s}$, overshoot $\sigma \% \approx 5 \%$. When the results of $\tau$ reduction, PID control rise time $t_{r} \approx 80 \mathrm{~s}$, regulation time $t_{s} \approx 170 \mathrm{~s}$, overshoot $\sigma \% \approx 10 \%$; NNPID control rise time $t_{r} \approx 120 \mathrm{~s}$, regulation time $t_{s} \approx 100 \mathrm{~s}$, overshoot $\sigma \% \approx 2 \%$.

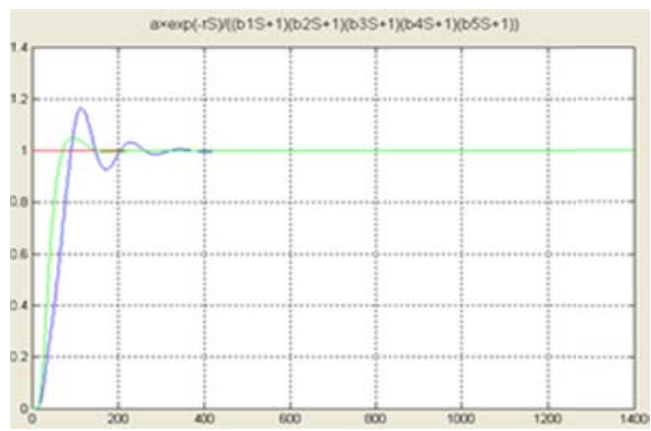

Fig. 8 When the results of the $\tau$ increase

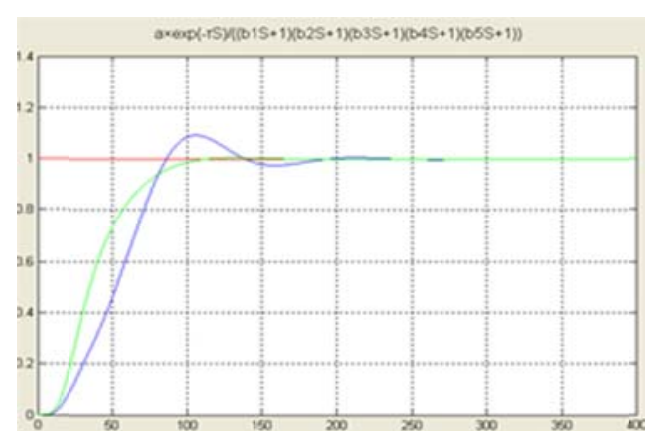

Fig.9 When the results of the $\tau$ reduction

From Fig.4 Fig.9, we can see that the PID controller is very sensitive to the parameters of the controlled object, while the BP neural network controller is not obvious to the parameters change.

Comparison of two Control Modes with Interference. In this paper, the amplitude of the random disturbance is $5 \%$ of the control signal $\boldsymbol{u}$. Simulation results after random interference as shown in Fig. 10.

The performance of resisting random interference of the two control modes is similar. This is due to the slow speed of the results of the neural network self-learning.

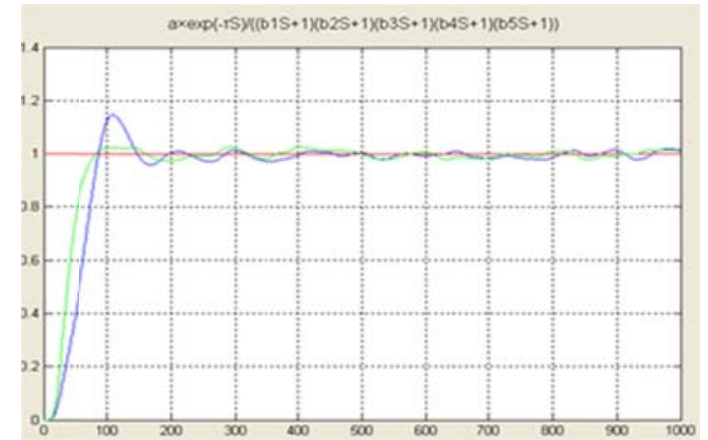

Fig.10 The result of random interference

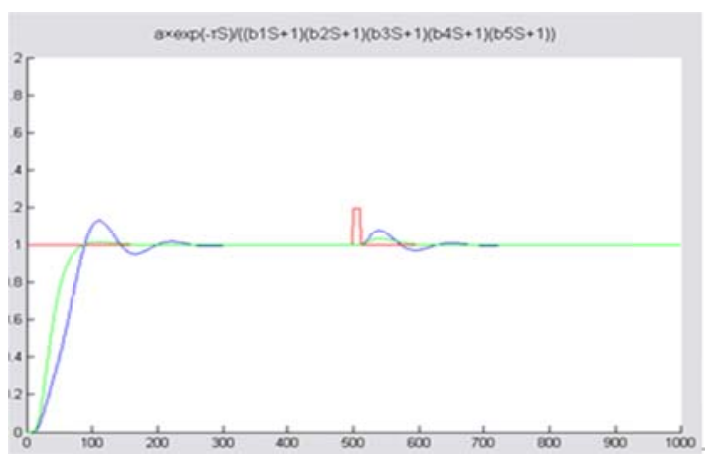

Fig. 11 The result of a given interference 
In this paper, the interference is amplitude 0.2 step signal, and the signal is added at 500th 510th time intervals. Used to compare two control modes for anti-jamming ability and recovery ability of given interference. Simulation results after given interference as shown in Fig. 11.

From the Fig.11, we can clearly see that the disturbance rejection capability of the neural network PID for a given disturbance is obviously superior to the traditional PID control.

\section{Conclusion}

In this paper, the PID control method based on BP neural network is proposed for the temperature control of PEMFC Stack, and in the test system are compared with the traditional PID control system. The design of the controller so that the system's overshoot, the Regulation time, the rise time and other performance indicators are within the allowable range, and more in line with the PEMFB Stack temperature control of this class of degeneration, long-hysteresis, uncertainty and nonlinear system with strong coupling.

\section{Acknowledgement}

Supported by Fundamental Research Funds for the Central Universities 06105053.

\section{References}

[1] Xuepeng Gao, Suang Cong. Comparative Study on Fast Learning Algorithms of BP Networks [J], Control and decision making, 2001.2:20-48.

[2] Dongri Sen, Shaohui Feng, Yijun Chen. Comparative Study on Fast Learning Algorithms of BP Networks[J], Chemical automation and Instrumentation, 2000(01):134-152.

[3] Dingyu Xue, Yangquan Chen. Technology and application of system simulation based on MATLAB/Simulink[M]. Beijing: Tsinghua University press,2002.

[4] P. Costamagna, S. Srinivasan. Quantum jumps in the PEMFC science and technology from the 1960s to the year 2000, Part II: Engineering, technology development and application aspects. Journal of Power Sources,2001. 102(1-2): 253-269.

[5] L.P.Jarvis, et al. Power assisted fuel cell. Journal of Power Sources 1998. 70(1-2)253-257.

[6] Jay T P,Anna G S, Huei P. Modeling and Control for PEM Fuel Cell Stack System[A]. 